\title{
La mise en ouvre des avantages exceptionnels dans la nouvelle législation algérienne de l'investissement
}

\begin{abstract}
:
Les changements opérés par la loi n¹6-09 du 3 août 2016 relative à la promotion de l'investissement ont substitué trois niveaux d'incitations aux deux régimes d'incitations qui existaient auparavant sous l'empire de l'ordonnance de 2001. Le niveau des avantages exceptionnels correspond d'une manière générale à l'ancien régime dérogatoire par la nature et la durée des avantages consentis, mais surtout par le procédé de sa mise en œuvre : la convention.
\end{abstract}

\section{Introduction :}

شملت التغييرات التي جاء بها القانون رقم 16-09 المؤرخ في3 أغسطس 2016 يتضمن ترقية الاستثمار عدة جوانب و من بينها استبدال نظامين الحوافز المتمثلين في النظام العام و النظام الإستثنائيان اللذان كانا قائمان سابقا في تشريع 2001 بثلاثة مستويات من المزايا . . و يقابل بشكل عام مستوى المزايا الاستثنائية لفائدة الاستثمارات ذات الأهمية الخاصة للاقتصاد الوطني النظام الاستثنائي السابق من حيث طبيعة ومدة المزايا، وبالأخص من حيث آدات تتفيذه و هي الاتفاقية.

Dr. Zouiten Abderrezak

Faculté de droit Université des frères Mentouri Constantine 2016 relative à la promotion de l'investissement publiée récemment ${ }^{(1)}$, est venue abroger dans leur quasi-totalité( ${ }^{(2)}$, les dispositions de l'ordonnance du 20 août 2001relative au développement de l'investissement ${ }^{(3)}$ qui faisait office jusque-là de code des investissements. Aux deux régimes d'incitation organisés par cette dernière, à savoir le régime général et le régime dérogatoire, ont succédé de nouveaux régimes prévus par 
la loi du 3 août 2016 relative à la promotion de l'investissement, et ce, en modifiant l'ordonnancement des avantages en trois niveaux d'incitations. Il s'agit en premier lieu, des avantages communs aux investissements éligibles, objet des articles 12,13et14. En second lieu, il s'agit des avantages supplémentaires au profit des activités privilégiées et/ou créatrices d'emplois, objet des articles 15 et 16 et enfin, des avantages exceptionnels au profit des investissements présentant un intérêt particulier pour l'économie nationale, objet des articles 17,18 et 19.

Si pour l'essentiel les investissements éligibles à ces avantages ont été reconduits par la nouvelle loi, il est à noter que l'article 5 de cette dernière exclut expressément les investissements de restructuration et de privatisation qui sous l'empire de l'ordonnance de 2001 étaient éligibles aux avantages. De même, les investissements réalisés dans le cadre de la concession et / ou de licence, qui étaient expressément cités dans l'article $1^{\text {er }}$ de l'ordonnance de 2001, semblent avoir été exclus du champ d'application de la nouvelle loi de 2016. Par contre, de nouvelles figures de l'investissement ont été introduites dans le champ d'application de la loi de 2016, à l'instar des investissements constitués par les biens, même ceux rénovés, constituant des apports extérieurs en nature entrant dans le cadre d'opérations de délocalisation d'activités à partir de l'étranger, et les biens faisant l'objet d'une levée d'option d'achat par le crédit preneur, dans le cadre du leasing international à la condition que ces biens soient introduits sur le territoire national à l'état neuf, selon l'article 6 .

La législation algérienne de l'investissement repose, et ce depuis le premier code d'inspiration libérale de $1993^{(4)}$, sur le principe de la graduation dans les avantages consentis. Cette graduation est consubstantielle de l'importance de l'investissement et de ses effets structurants sur l'économie nationale. Cette équation constitue le fondement même des niveaux des avantages supplémentaires et des avantages exceptionnels de la loi de 2016. Ces niveaux reposent sur le postulat selon lequel, plus l'intérêt de l'investissement est grand et plus les avantages qui pourraient lui être consentis le seraient aussi. Aussi, la différence fondamentale qui existe entre ces niveaux, qui s'apparentent du reste à des régimes supérieurs, spécialement celui des avantages exceptionnels, et le niveau des avantages communs, réside dans la nature, la durée et le contenu des avantages consentis.

Des trois niveaux cités plus haut, celui qui intéresse notre présente étude est celui des avantages exceptionnels au profit des investissements présentant un intérêt particulier pour l'économie nationale, et ce, par la singularité du procédé utilisé pour sa mise en œuvre : celui de la convention. Cette dernière consacre une contractualisation de la relation d'investissement entre l'Etat et l'investisseur.

Aussi, pour une bonne compréhension de la question, nous procéderons dans un premier temps à un essai de délimitation de la notion d'investissement 


\section{La mise en œuvre des avantages exceptionnels dans la nouvelle législation algérienne de l'investissement}

présentant un intérêt particulier pour l'économie nationale qui reste assez imprécise et compte tenu du fait que ces derniers constituent le soubassement du régime de la convention (1). Dans un second temps, nous mettrons en exergue la place de la convention dans la mise en œuvre de ce régime d'avantages exceptionnels (2).

$1-:$ Les critères qui fondent le bénéfice des avantages exceptionnels

Les investissements présentant un intérêt particulier pour l'économie nationale et le régime de la convention trouvent leur siège dans l'article 17 de la loi de 2016 précitée qui constitue de ce fait le fondement légal de cette notion en droit algérien. Cet article 17 dispose : « Bénéficient des avantages exceptionnel établis par voie de convention négociée entre l'investisseur et l'agence agissant pour le compte de l'Etat, les investissements présentant un intérêt particulier pour l'économie nationale.

La convention est conclue par l'agence, après approbation du conseil national de l'investissement.

Les critères de qualification des investissements visés à l'alinéa 1 ci-dessus, ainsi que le contenu et les procédures de traitement du dossier de demande de bénéfice des avantages exceptionnels sont fixés par voie réglementaire. "

Cette disposition n'a pas apporté des éléments de réponse clairs et précis en matière de délimitation et de définition des investissements présentant un intérêt particulier pour l'économie nationale. L'alinéa 3 de l'article en question s'est contenté de laisser au règlement le soin de fixer ultérieurement les critères de qualification de ces investissements présentant un intérêt particulier. Autant dire dés le début, que ce texte d'application peut ne jamais voir le jour. Nous en voulons pour preuve que cette même disposition était prévue par l'article 10 de l'ordonnance 01-03 du 20 août 2001 relative au développement de l'investissement, pour le même type d'investissements et pour le même type d'avantages. Aucun texte réglementaire relatif à la fixation de critères de qualification des investissements visés plus-haut, n'a été pris pendant toute la durée d'application de l'ordonnance, c'est-à-dire de 2001 à 2016.

1-1: La reconduction probable du diptyque intérêt économique / intérêt écologique

Contrairement aux législations qui l'ont précédé, la loi du 3 août 2016 relative à la promotion de l'investissement est muette sur cette question des critères de l'intérêt particulier du projet d'investissement. A titre d'illustration, l'article 15 du décret législatif ${ }^{\circ} 93-12$ du 5 octobre 1993 relatif à la promotion de l'investissement retenait les critères de la dimension du projet, de la technologie utilisée, du taux d'intégration de la production développée et enfin des gains en devises et la rentabilité à long terme du projet. De son côté, l'ordonnance du 20 août 2001relative au développement de l'investissement avait retenu, en son article 10 alinéa 2 , au moins en ce qui concerne les 
investissements présentant un intérêt écologique, un certain nombre de critères concernant l'utilisation des technologies propres susceptibles de préserver l'environnement, la protection des ressources naturelles, l'économie d'énergie et devant conduire au développement durable. Ceci nous avait donné déjà quelques clés de lecture concernant les critères de qualification des investissements présentant un intérêt particulier pour l'économie nationale. Aussi, devant le silence de la loi, et dans l'attente d'un texte réglementaire incertain, il nous semble que les critères d'éligibilité au régime des avantages exceptionnels vont probablement continuer à s'articuler autour du diptyque que nous avait suggéré auparavant l'alinéa 2 de l'article 10 de l'ordonnance de 2001, selon lequel l'intérêt particulier peut se confondre soit avec l'intérêt écologique, soit avec l'intérêt économique stricto-sensu.

Le premier volet du diptyque concerne les investissements présentant un intérêt économique stricto-sensu. Il peut être assimilé d'une façon générale, à ce qu'il est convenu d'appeler un projet structurant. En règle générale, ce dernier constitue le plus souvent un pôle de développement économique, social, et spatial. Il est également générateur d'un nombre assez élevé d'emplois. Il donne lieu également à des investissements importants en termes financiers. Enfin, il constitue un vecteur d'apport de technologies nouvelles. Pour les besoins de la démonstration, nous allons prendre appui sur certains de ces critères que nous venons d'énumérer.

Le projet d'investissement doit être un pôle de développement économique, social et spatial. Il doit constituer pour la localité dans laquelle il est mis en œuvre un instrument d'entrainement vers le progrès économique et social. Sa mise en œuvre doit avoir vocation à entrainer la création de richesses et d'emplois, concourir au développement d'un réseau de sous-traitants ou d'activités connexes et à l'essor des activités créatrices de valeur ajoutée. Dans cette projection spatiale, il faudrait exclure les investissements qui bénéficient d'avantages spécifiques liés au régime prévu par l'article 13 de la loi de 2016 relative à la promotion de l'investissement concernant les investissements réalisés dans les localités relevant du Sud et des Hauts-Plateaux ainsi que dans toute autre zone dont le développement nécessite une contribution particulière de l'Etat.

Afin de présenter un intérêt économique évident, l'investissement, doit en deuxième lieu, être générateur d'emplois. A cet égard, le nombre et la qualité des emplois créés seront vus comme un élément d'appréciation du caractère structurant du projet. Cet aspect concerne aussi bien les emplois permanents au sein de l'entreprise, que ceux générés par les activités connexes du projet (sous-traitants, etc.). En l'absence de textes de référence précis sur les critères de l'importance économique du projet d'investissement, et spécialement en matière d'emplois créés, le législateur algérien a tout de même laisser apparaitre un indice quantitatif pouvant dévoiler quelque peu son 


\section{La mise en œuvre des avantages exceptionnels dans la nouvelle législation algérienne de l'investissement}

intention sur le nombre d'emplois générés par l'investissement afin qu'il prétende au régime des avantages exceptionnels. Seulement, cette référence ne concerne pas spécifiquement ce dernier. L'article 16 de la loi de 2016 relatif aux avantages supplémentaires au profit des activités privilégiées et/ou créatrices d'emplois fait bénéficier les investissements créant plus de cent (100) emplois permanents, d'un allongement de trois (3) à cinq (5) ans dans la durée des avantages d'exploitation consentis au profit des investissements réalisés durant la période allant de la date d'enregistrement de l'investissement à l'achèvement de la première année de la phase d'exploitation. A contrario, nous pouvons déduire que les investissements éligibles au régime des avantages exceptionnels devraient créer un nombre d'emplois encore plus important, du seul fait que le régime des avantages exceptionnels est supérieur à celui des avantages supplémentaires en ce que la durée des avantages d'exploitation est portée à dix (10) ans. Ce critère quantitatif de cent (100) emplois, constitue donc une base de référence qui nous permet d'avoir une représentation pertinente de ce critère de la création d'emplois.

En troisième lieu, le critère financier est également déterminant dans l'importance économique de l'investissement. Le projet doit donner lieu à des investissements importants en termes financiers. Le montant total des immobilisations financières liées à un projet structurant dans sa phase de construction doit au moins être égal ou supérieur à un seuil qui est censé être défini par le conseil national de l'investissement (CNI). A défaut de cela, et dans l'attente de la publication d'un texte réglementaire sur les critères de qualification de l'importance économique, sous réserve encore une fois de sa publication, nous nous remettons à d'autres dispositions légales pour cerner un tant soit peu ce critère. En effet, l'article 14 de la loi de 2016 a proposé un seuil de montant au-delà duquel les investissements doivent être soumis à l'accord préalable du C.N.I. ${ }^{(5)}$ Quel sens donner à cette disposition ?

Cet article 14 pose un critère financier de détermination des investissements soumis au principe de l'automaticité du bénéfice des avantages communs à tous les investissements qui satisfassent aux conditions posées par l'article 4 de la loi de 2016 et notamment leur enregistrement auprès de l'agence nationale de développement de l'investissement (A.N.D.I). Cette agence est habilitée par les termes mêmes de $1 \mathrm{a} \operatorname{loi}^{\left({ }^{(}\right)}$à faire bénéficier de manière automatique, des avantages de réalisation prévus par la présente loi, les investissements ne figurant pas sur les listes négatives. A contrario, ce seuil nous permet également d'identifier les investissements soumis à l'appréciation discrétionnaire du C.N.I, dont font parties les investissements éligibles aux avantages exceptionnels du fait de leur importance économique. A notre sens, et au-delà de 5.000.000.000 DA le projet devra basculer logiquement dans le régime des avantages exceptionnels puisque il est exigé l'accord préalable du 
C.N.I à sa réalisation. Par déduction, et toujours dans l'attente des textes d'application de la loi de 2016, il nous semble que le critère financier de qualification de l'importance économique va s'articuler précisément sur le seuil dégagé par l'article 14 de la loi de 2016. Il sera donc exigé, pour le bénéfice des avantages exceptionnels que le montant financier soit supérieur au seuil cité précédemment.

Le deuxième volet du diptyque concerne les investissements présentant un intérêt écologique. Il nous parait très probable que le législateur fera bénéficier ces investissements d'un statut spécial en les intégrant dans la catégorie des investissements présentant un intérêt particulier pour l'économie nationale et ce, au regard de la place grandissante des préoccupations environnementales dans les politiques publiques. Il est regrettable que le législateur de 2016 n'ait pas avancé certaines pistes pouvant identifier les investissements dont l'intérêt écologique serait avéré, contrairement à celui de 2001.

Avant toute chose, il nous faudra signaler, qu'au-delà des avantages exceptionnels dont peuvent bénéficier les investissements présentant un intérêt écologique, l'article 3 de la loi no $16-09$ du 3 août 2016 relative à la promotion de l'investissement renferme une préconisation générale pour le respect par les investissements de la protection de l'environnement. Seulement, cette préconisation peut s'avérer insuffisante dans la prise en charge des exigences écologiques par les investisseurs. Pour cela, l'accompagnement de cette préconisation par un régime d'incitations spécifique s'impose de lui-même.

Aussi, il nous parait logique que le législateur se basera, comme cela a été le cas auparavant avec l'alinéa 1 de l'article 10 de l'ordonnance de 2001 sur les critères de l'utilisation des technologies propres susceptibles de préserver l'environnement, la protection des ressources naturelles, l'économie d'énergie conduisant au développement durable.

Le traitement privilégié fait d'avantages spéciaux aux investissements émanant des entreprises locales, mais surtout étrangères qui font l'effort de s'intégrer dans les nouveaux concepts qui ont conduit à l'émergence de la notion d'investissement socialement responsable (ISR), peut être réalisé dans le cadre d'une convention négociée et conclue avec l'A.N.D.I pour le compte de l'Etat.

Aussi, et par-delà les mesures d'autorité mises en place par la législation et la réglementation internes sur l'environnement mettant en œuvre des normes environnementales ${ }^{(7)}$, le droit algérien s'inscrit avant toute chose dans le sillage des conventions et accords internationaux de préservation de l'environnement, même s'il faut d'ores et déjà souligné leur caractère juridiquement souvent non contraignant. Le dernier accord paraphé puis ratifié par l'Algérie a été l'accord de Paris sur les changements climatiques ${ }^{(8)}$. 


\section{La mise en œuvre des avantages exceptionnels dans la nouvelle législation algérienne de l'investissement}

La relation entre la croissance économique et la préservation de l'environnement, qui a toujours suscité un débat conflictuel, s'est beaucoup altérée avec le développement vertigineux des sciences et des technologies. La recherche effrénée de la croissance s'est souvent réalisée sur la base de modèles d'exploitation intensive des ressources naturelles. Par-delà cet aspect, les industries, qui dans leur essence, devaient permettre l'amélioration de la qualité de vie de l'homme, se trouvent être paradoxalement à l'origine de rejets souvent toxiques qui sont venus menacer toujours un peu plus l'état déjà fragile de l'environnement.

Aussi, le niveau des avantages exceptionnels qui se base sur la pédagogie des avantages spéciaux devra consacrer une démarche, celle de la négociation d'avantages dès lors que l'investissement projeté donnera des gages sérieux de conciliation de la nécessité de la préservation de l'environnement et la poursuite de l'objectif de croissance économique et du profit qui sous-tend l'action de l'investisseur.

A titre d'illustration, l'activité de dessalement de l'eau de mer est un des domaines de prédilection de l'octroi d'avantages exceptionnels pour les investissements présentant un intérêt écologique dans le cadre des conventions d'investissement. Cet intérêt se manifeste avant toute chose dans les technologies utilisées, mais également dans la production d'une denrée qui tend à se raréfier non seulement en Algérie, mais sur l'ensemble du pourtour méditerranéen.

Dans ce contexte, Le CNI a par le passé, approuvé un certain nombre de projets de conventions présentés par l'ANDI. Ces projets concernaient la réalisation d'unités de dessalement d'eau de $\operatorname{mer}^{(9)}$.

Le procédé de dessalement de l'eau de mer impacte nettement l'environnement. Pour cette raison, les pouvoirs publics tiennent à accompagner la construction de ces unités dans le cadre de la convention d'investissement afin de soumettre les investisseurs à des obligations contractuelles strictes pour la protection de l'environnent.

1-2: L'instance chargée de la fixation des critères de l'intérêt particulier pour l'économie nationale

Institué par l'article 18 de l'ordonnance $n^{\circ} 01-03$ du 20 août 2001 relative au développement de l'investissement, le CNI constitue l'échelon stratégique en matière d'organes de l'investissement. En abrogeant les dispositions de l'ordonnance de 2001, la loi n ${ }^{\circ} 16-09$ du 3 août 2016 relative à la promotion de l'investissement, en son article 37, a maintenu au titre des exceptions, les dispositions des articles 6,18 et 22 relatives aux deux organes principaux de la chaîne d'investissement que sont le C.N.I et l'ANDI. 
D'une manière générale, le CNI est un organe politique qui symbolise le niveau stratégique du dispositif. Il représente l'Etat et cristallise tout le poids de l'instance politique dans la définition et la mise en œuvre des politiques publiques en matière d'investissement. Il a pour mission de définir les orientations gouvernementales en la matière et il a compétence à agréer les investissements qui lui semblent utiles au développement économique du pays. Il en résulte que ce nouvel organe se soit saisi d'un champ de décision très étendu et relègue de ce fait l'agence, c'est à dire l'ANDI, à un rôle auxiliaire. Il constitue de ce fait, le maillon essentiel de la politique nationale de l'investissement.

Créé auprès du ministre en charge de l'investissement, ce conseil est placé sous l'autorité du chef du gouvernement qui en assure la présidence et ce, en vertu de l'article 18 de l'ordonnance $n^{\circ} 01-03$ du 20 août $2001^{(10)}$ et de l'article $12 \mathrm{du}$ décret exécutif $\mathrm{n}^{\circ} 06-355$ du 9 octobre 2006 relatif à sa composition, à son organisation et à son fonctionnement ${ }^{(11)}$. Le CNI est composé de représentants de ministères de souveraineté et techniques pouvant intervenir dans la chaîne de l'investissement. Le secrétariat du conseil est assuré par l'ANDI. Le président du conseil d'administration et le directeur général de cette agence peuvent assister en tant qu'observateurs aux réunions du conseil.

Les missions du CNI sont considérables. Elles découlent d'un pouvoir de décision très étendu et ce, à travers notamment la mission d'approuver ou non les conventions pouvant être conclues avec des investisseurs dans des projets d'envergure nationale.

En matière de détermination des critères de qualification des investissements présentant un intérêt particulier pour l'économie nationale, le C.N.I devra continuer à exercer le rôle prépondérant qui était le sien par le passé. En effet, dans tous les cas de figure, nous estimons qu'il reviendra au C.N.I de définir ces critères. Dans la première hypothèse où un texte réglementaire sera pris pour définir les critères de l'intérêt particulier, celui-ci se fera nécessairement sur la base de travaux préparatoires, recommandations ou décision émanant du C.N.I lui-même. Dans la seconde hypothèse, le texte réglementaire ne sera pas pris et nous serions alors dans la configuration que nous avions connue de 2001 à 2016, où, en l'absence de celui-ci, le C.N.I exerçait un rôle discrétionnaire dans l'appréciation de l'importance économique de l'investissement afin de faire bénéficier ce dernier du régime de la convention. De plus, sur la base de l'article 17 de la loi de 2016, qui prévoit que la convention est conclue par l'agence après approbation du conseil national de l'investissement, nous considérons que la décision ultime en matière d'octroi d'avantages dans le cadre de la convention d'investissement sera celle que rendra le CNI, abstraction faite du projet de décision qu'a pu prendre l'ANDI à ce propos. Cette dernière devra donc soumettre à 


\section{La mise en œuvre des avantages exceptionnels dans la nouvelle législation algérienne de l'investissement}

l'approbation du CNI tout projet de décision d'octroi d'avantages à charge pour ce dernier de l'approuver ou de le révoquer. Cette observation nous permet de conclure que l'ultime et la seule décision sera celle du CNI, qui à travers son examen des projets d'investissements qui doivent lui être soumis, décidera en toute discrétion, des critères applicables aux investissements, cas par cas, afin de les rendre éligibles au régime de la convention d'investissement. Le rôle de cet organe est par voie de conséquence, prééminent. Nous avions déjà souligné que le texte réglementaire peut ne jamais voir le jour. C'est à se demander si cet état de fait ne serait pas délibéré afin de laisser au CNI un champ de compétence assez élargi.

2-: Régime et nature de la convention d'investissement comme instrument de mise en œuvre des avantages exceptionnels

La convention d'investissement participe d'une gestion partenariale, concertée de l'investissement en associant l'investisseur à définir les termes mêmes de l'accord qu'il compte conclure avec l'Etat. Cette pratique nouvelle, fait reculer la sphère d'impérativité du droit de l'investissement ${ }^{(12)}$, par l'élargissement des marges de négociation en consacrant le contrat comme instrument de réalisation de l'investissement.

La convention prévue par l'article 17 de la loi de 2016 précitée, permet d'accéder à un régime supérieur d'avantages puisque l'investisseur peut négocier avec l'agence agissant pour le compte de l'Etat, des avantages qui peuvent aller au-delà de ce que prévoit le code des investissements. Il y aurait tout de même une réserve à tout cela, celle d'accorder ces avantages conformément à la législation en vigueur, d'après les termes de l'article 18 de la loi de 2016, paragraphe $b$.

La convention renvoie donc à une pratique courante dans les relations économiques internationales, qui a était sujette à débats pour un long moment. Il s'agit de la notion de contrats d'investissement conclus entre les Etats en développement et des sujets de droit privé qu'ils soient individus ou personnes morales, c'est-à-dire des firmes étrangères. Ces contrats d'investissement, que certains nomment également contrats $\mathrm{d}^{\prime} \mathrm{Etat}^{(13)}$, par référence à une notion d'origine anglo-saxonne, les «states contrats», sont une invention de la doctrine et de la pratique arbitrale afin d'internationaliser les contrats suite aux énormes problèmes qui ont surgi dans les années 1950-1960 entre les Etats et les concessionnaires pétroliers. Sans préjuger de sa nature juridique que l'on examiner par la suite, le législateur algérien s'est simplement contenté de l'utilisation du vocable convention.

2-1: Le régime de la convention d'investissement

Dans ce cadre, il convient de s'interroger sur les conditions légales exigées par la loi pour la validité de la convention et le contenu et les procédures de traitement du dossier de demande de bénéfice des avantages exceptionnels et 
ce, en ayant à l'esprit que ces derniers tout autant que les critères n'ont pas été définis par la loi de 2016 et que celle-ci s'en remet à un règlement ultérieur.

En premier lieu, la convention d'investissement requiert la réunion de conditions posées par l'article 17 de la loi de 2016 relative à la promotion de l'investissement. Ces conditions tiennent aussi bien à la forme qu'au fond.

En matière de conditions de validité tenant à la forme, il convient d'ores et déjà de souligner que la loi de 2016 consacre un recul par rapport à l'ordonnance de 2001. En effet, la condition de publication de la convention au journal officiel de la République Algérienne a été supprimée. Ce recul ne nous semble pas justifié même si la pratique antérieure qui rendait obligatoire la publication de la convention au journal officiel n'a pas été assez convaincante du point de vue de la transparence dans les accords passés avec les investisseurs $^{(14)}$. De plus, l'acte solennel pris sous la forme d'un décret exécutif d'approbation de la convention d'investissement donne tout son sens à la notion d'engagement contractuel de l'Etat.

Aussi, la seule condition de forme qui reste exigible, c'est celle de la conclusion de la convention par l'agence, c'est à dire L'A.N.D.I, après approbation du conseil national de l'investissement. Or, cette condition réaffirme le rôle prééminent du C.N.I que nous avons souligné auparavant et en même temps, cette condition se confond avec la procédure de traitement du dossier de demande des avantages exceptionnels sous le régime de la convention que nous examinerons plus loin.

La condition de fond pour la validité de la convention d'investissement se réfère à l'intérêt particulier que présente l'investissement pour l'économie nationale. Ce dernier, comme nous l'avons examiné auparavant, est circonscrit aux investissements de nature à présenter un avantage écologique ou économique évident. Dans l'attente d'un texte réglementaire, les critères retenus pour l'appréciation de cette importance sont laissés, comme nous l'avons vu précédemment, à la seule discrétion du CNI, qui les apprécie lors de l'examen des procédures de demande d'avantages exceptionnels dans le régime de la convention. L'imprécision qui entoure cette notion d'intérêt particulier pour l'économie nationale rend la gestion du dispositif d'octroi d'avantages dans le cadre du régime de la convention, assez «opaque et subjectif» dans la sélection des projets, pour reprendre l'expression de M. Haroun ${ }^{(15)}$. L'exigence de la conformité avec la législation en vigueur vient se greffer à la condition de l'intérêt particulier pour l'économie nationale. Cette condition peut paraitre contradictoire avec la vocation même du régime des avantages exceptionnels. Cette dernière repose sur l'octroi d'avantages qui sont, par principe, supérieurs à ceux prévus par la législation en vigueur sur la base de laquelle ils sont définis. Si ces avantages peuvent aller au-delà de ce que prévoit la législation sur les investissements, ils ne peuvent, par contre, aller à l'encontre de la loi. 


\section{La mise en ouvre des avantages exceptionnels dans la nouvelle législation algérienne de l'investissement}

Aussi, de ce qui précède, nous estimons, que la seule condition de fond exigible en matière de validité de la convention d'investissement, concerne le caractère particulièrement important de l'investissement projeté, en termes d'apports multiformes que ce dernier pourra présenter à l'économie nationale selon les critères écologiques et économiques et que nous avons identifiés auparavant.

En second lieu, et concernant la procédure de traitement du dossier de demande des avantages exceptionnels sous le régime de la convention, et dans l'attente du texte réglementaire régissant cette question, cette dernière devra être conduite selon le schéma institutionnel qui a prévalu jusqu'à maintenant. Elle est négociée sous la conduite du ministre chargé de la promotion des investissements. La négociation, qui constitue l'ensemble des actes préalables à la conclusion du contrat, a pour finalité de préciser et de résoudre les problèmes qui pourraient surgir dans la mise en œuvre des engagements contractuels des parties. Dans cette négociation, la présence de l'Etat ou l'une de ses émanations est un préalable indispensable à l'existence de cet instrument. Cette faculté reconnue au ministre découle de ses prérogatives générales puisqu'il a pour mission de proposer, puis de suivre la mise en œuvre de la politique et des stratégies de promotion des investissements. Elle découle ensuite de sa qualité d'autorité de rattachement du CNI qui est créé auprès du ministre en charge de l'investissement, bien que ce conseil soit placé sous l'autorité du chef du gouvernement qui en assure la présidence et ce, en vertu de l'article 18 de l'ordonnance $\mathrm{n}^{\circ} 01-03$ et de l'article 12 du décret exécutif $\mathrm{n}^{\circ}$ 06-355 du 9 octobre 2006 relatif à sa composition, à son organisation et à son fonctionnement. En pratique, la négociation de la convention est menée par l'ANDI, agissant pour le compte de l'Etat, qui compte en son sein une division spécialement créée pour la gestion des grands projets d'investissements. Le rôle prépondérant du CNI, que nous avons évoqué auparavant prend en matière de convention tout son sens. Après que l'investisseur ait exprimé son intention de bénéficier du régime de la convention, le CNI doit avant toute chose donner son aval pour rendre cet investissement éligible au régime des avantages exceptionnels. La division des grands projets de l'ANDI, sous le contrôle du ministre chargé de l'investissement, négocie les avantages à octroyer aux investissements présentant un intérêt particulier pour l'économie nationale. Il est évident que le ministre en charge de l'investissement doit se référer constamment au président du CNI, en l'occurrence le chef du gouvernement. Le projet de convention est par la suite transmis à cette instance politique qui approuve par résolution prise en session, la conclusion de cette convention. Cette dernière ne peut être effective qu'après autorisation expresse émanant du ministre en charge de l'investissement enjoignant au directeur général de l'ANDI, de procéder à la signature de la convention d'investissement. 
2-2: La nature controversée de la convention

De prime abord, il est à souligner les réticences du législateur algérien à s'inscrire dans la controverse de la qualification juridique de la convention prévue à l'article 17 de la loi de 2016 relative à la promotion de l'investissement. Nulle part dans ce texte de loi, nous ne trouvons une autre appellation que celle de convention. Alors que les accords conclus en matière de coopération économique internationale sont souvent qualifiés de conventions d'investissement ou d'établissement, de contrats d'investissement ou encore d'accords de développement économique, le législateur algérien a pris le soin de ne pas se projeter dans un débat qui a surtout été l'œuvre de la doctrine juridique des pays développés à un moment où les firmes des pays développés voulaient soustraire ces conventions à la compétence du droit interne, c'est à dire le droit de territorialité de l'investissement . L'essentiel dans la démarche du législateur algérien a été de mettre en place un régime conventionnel qui permette à l'investisseur aussi bien local qu'étranger de négocier les conditions de la réalisation de son investissement tant sur le plan des garanties de la sécurité juridique dues à l'opération d'investissement qu'aux avantages dont il peut bénéficier.

Il y a lieu de distinguer deux situations possibles dans lesquelles la convention prévue par l'article 17 de l'ordonnance de 2016 relative à la promotion de l'investissement peut être assimilée soit à un contrat de droit interne, soit à un contrat de droit international. L'avènement de la notion de contrats d'Etat, qui constitue le référent théorique de la convention et qui a eu les faveurs d'une bonne partie de la doctrine des pays développés à la fin du siècle qui vient de s'écouler, avait pour objectif non dissimulé la protection des étrangers contre la partialité supposée des juges nationaux et le recours démesuré aux prérogatives de souveraineté de l'Etat d'accueil.

Dans la première hypothèse, celle de la conclusion de cette convention entre l'A.N.D.I et un investisseur local, la nature juridique de la convention d'investissement ne souffre d'aucune ambiguïté possible. Elle constitue un contrat de droit interne relevant de la catégorie des contrats administratifs. La qualification de contrat administratif provient du seul critère organique consacré par le droit administratif algérien en application de l'article 800 de la loi $\mathrm{n}^{\circ}$ 08-09 du 25 février 2008 portant code de procédure civile et administrative $^{(16)}$ qui définit le litige administratif . De par ses statuts, l'ANDI, qui conclut pour le compte de l'Etat les conventions d'investissement, jouissant de la personnalité administrative, en tant qu'établissement public à caractère administratif, les contrats conclus par elle, ont donc la qualité de contrats administratifs dont les réclamations sur leur interprétation ou exécution ressortent de la compétence du droit interne et spécialement de la juridiction administrative. 


\section{La mise en ouvre des avantages exceptionnels dans la nouvelle législation algérienne de l'investissement}

Dans la seconde hypothèse, la convention est conclue avec un investisseur étranger. Peut-on dés lors la qualifier de contrat d'Etat et sa nature juridique serait- elle celle d'un contrat de droit international ?

Pour avoir un temps dominé le débat juridique en matière de droit international économique, la problématique des contrats d'investissement, qualifiés d'Etat, avait mis en présence deux thèses contradictoires. Les pays en développement, c'est-à-dire les Etats d'accueil des investissements exprimaient en général, leur préférence pour l'ordre interne, alors que les investisseurs étrangers et leurs Etats, les pays développés, préféraient se soumettre à un ordre différent : celui de l'ordre international. Les développements récents font part du dépassement de cette notion dont l'unique intérêt reposait, semble-t-il, sur sa capacité à internationaliser le droit de l'investissement. Or, ce mouvement a été réalisé pour un certain nombre d'auteurs ${ }^{(17)}$ par la multiplication des conventions multilatérales et surtout bilatérales de protection des investissements ${ }^{(18)}$. Aussi, nous estimons que la convention d'investissement régie par le droit algérien nous parait se focaliser surtout sur les avantages consentis aux investisseurs, même si, ici et là, des garanties sont réitérées dans certaines de ses stipulations. A partir de là, le contentieux qui pourra naître entre l'Etat et l'investisseur étranger va concerner l'exécution d'obligations dont l'impact sera surtout interne, auxquelles l'Etat a souscrit en accordant des défiscalisations et autres avantages. De fait, cette convention nous semble adossée dans sa quasi- totalité au droit interne, et ce, malgré l'existence possible d'éléments d'extranéité, telle que la clause d'arbitrage.

\section{Conclusion}

Dans l'attente du parachèvement du dispositif juridique de l'investissement avec la promulgation des textes d'application de la loi n⿳亠口冋-09 du 3 août 2016 relative à la promotion de l'investissement, nous ne pouvons au stade actuel des choses porter une appréciation sur les avancées ou au contraire les régressions que cette loi comporte en matière de régime d'avantages exceptionnels et la convention comme instrument de sa mise en œuvre. Seulement, il nous parait que le décloisonnement opéré entre les niveaux d'avantages que vient de consacrer la nouvelle législation peut entrainer une généralisation voire une banalisation du procédé de la convention qui peut avoir pour conséquence de rendre la notion d'intérêt particulier inintelligible. Ceci étant et puisque le principe cardinal qui semble être celui de ce dispositif consiste à faire bénéficier l'investissement de l'incitation la plus avantageuse en cas de coexistence d'avantages de même nature, selon les termes de l'article 19 de la loi de 2016, ce principe aussi généreux soit-il nous semble procéder à un 
mélange de genre qui risque de dévier cette réforme de son objectif premier, celui de la simplification.

\section{Notes}

1-J.o.r.a n ${ }^{\circ} 46$ du 3 Août 2016.

2-A l'exception des articles 6,18 et 22 , qui ont trait aux organes de l'investissement, à savoir, l'agence nationale de développement de l'investissement (A.N.D.I) et le conseil national de l'investissement (C.N.I).

3 -J.o.r.a n 47 du 22 Août 2001

4 -Décret législatif $n^{\circ} 93-12$ du 5 octobre 1993 relatif à la promotion de l'investissement. J.O.R.A n ${ }^{\circ} 64$ du 10 octobre 1993

5- Cet article dispose : «Nonobstant les dispositions de l'article 8 ci-dessus, l'octroi des avantages aux investissements dont le montant est égal ou supérieur à cinq milliards de dinars (5.000.000.000 DA) est soumis à l'accord préalable du conseil national de l'investissement visé à l'article 18 de l'ordonnance $n^{\circ} 01-03 \ldots . "$

6- Voir à ce sujet l'article 4 de la loi de 2016 relative à la promotion de l'investissement.

7 -Celles-ci sont représentées essentiellement par la loi $n^{\circ}$ 03-03 du 19 juillet 2003 relative à la protection de l'environnement dans le cadre du développement durable, (J.o.r.a $\mathrm{n}^{\circ} 43$ du 20 juillet 2003) et le décret exécutif $\mathrm{n}^{\circ}$ 06-198 du 4 juin 2006 définissant la réglementation applicable aux établissements classées pour la protection de l'environnement (J.o.r.a n ${ }^{\circ} 37 \mathrm{du} 4$ juin 2006).

8 -La conférence de Paris sur le climat (Cop 21), tenue du 30 novembre au 12 décembre 2015, a abouti à un accord international sur le climat applicable à tous les pays participants, dont l'Algérie, fixant comme objectif une limitation du réchauffement climatique mondial entre 1,5 et $2^{\circ}$ d'ici 2100 . L'Algérie a ratifié cet accord par le décret présidentiel $\mathrm{n}^{\circ} 16-262$ du 13 octobre 2016. (J.o.r.a ${ }^{\circ} 60 \mathrm{du} 13$ octobre 2016).

9 -Une convention conclue entre l'ANDI et miyah Tipaza Spa, signée le $25 / 06 / 2008$, portait sur la réalisation d'une usine de dessalement d'eau de mer d'une capacité nominale de $120000 \mathrm{~m} 3 /$ jour, située à Fouka, Wilaya de Tipaza, in, revue éditée par l'ANDI, «ANDI news » bulletin trimestriel, Août $2008 \mathrm{~N}^{\circ} 06$.

10 -A titre de rappel cet article n'a pas été abrogé par la loi n ${ }^{\circ} 16-09$ du 3 août 2016 relative à la promotion de l'investissement.

11 -J.o.r.a n 64 du 11 octobre 2006.

12 - Cette idée a été défendue par A. Bencheneb : In, «La notion d'avantages supplémentaires dans le droit des investissements: l'exemple algérien ». R.D.A.I/IBLJ, n³, 1999. 


\section{La mise en œuvre des avantages exceptionnels dans la nouvelle législation algérienne de l'investissement}

13- Voir à ce propos, Charles Leben, "Quelques réflexions théoriques à propos des contrats d'Etat», in, Souveraineté étatique et marchés internationaux. A propos de 30 ans de recherche du CREDIMI. Mélanges en l'honneur de Philippe Kahn. Litec. Année 2000- Volume 20.

14 - A l'exception de la première convention conclue du temps de l'A.P.S.I pour le compte de l'Etat algérien et le groupe égyptien Orascom (J.o.r.a $n^{\circ} 80$ du 26 décembre 2001, p, 8), les décrets exécutifs d'approbation des conventions d'investissement se limitaient simplement à approuver les conventions conclues sans aucune autre précision sur la nature des avantages consentis. Or, la transparence appropriée voudrait que les avantages consentis souvent fiscaux, soient portés à la connaissance du public, car ils constituent un manque à gagner pour le trésor public.

15- In, " Le régime des investissements en Algérie, à la lumière des conventions franco-algériennes »Litec. Paris. 2000, p 344. Cette analyse, fautil le rappeler, avait pour base le décret législatif $n^{\circ} 93-12$ du 5 octobre 1993 relatif à la promotion de l'investissement. Le régime de la convention prévu par cette législation a été repris dans sa quasi-intégralité par l'ordonnance de 2001 relative au développement de l'investissement, puis par la loi de 2016.

16- J.o.r.a $\mathrm{n}^{\circ} 21 \mathrm{du} 23$ avril 2008.

17- Cette thèse du déclin des contrats d'Etat au profit des conventions internationales est parfaitement illustrée par les opinions émises par D. Carreau et P. Juillard in : " Droit international économique », 3éme édition, Dalloz, Paris, 2007, p. 491.

18 -L'apparition dans les années 60 puis le développement des conventions ou Traités bilatéraux sur les investissements dans les années $70-80$ ont éclipsé la question des contrats d'Etat, puisque ces derniers sont souvent placés sous la protection des premiers. Ceci a donné lieu à l'apparition d'une clause célèbre en droit des investissements appelée la clause parapluie ou « umbrella clause », en vertu de laquelle les contrats d'investissements sont sous le parapluie des Traités bilatéraux d'investissement.

\section{Références bibliographiques}

Bekhechi Mohamed Abdelwahab: Quelques éléments de réflexion sur la pratique algérienne du contrat d'Etat, in les contrats internationaux et Pays en voie de développement sous la direction de H. CASSAN, économica, Paris, 1989.

Bencheneb Ali : La notion d'avantages supplémentaires dans le droit des investissements: l'exemple algérien, Revue de Droit des Affaires Internationales (R.D.A.I)/IBLJ, n³, 1999.

D. Carreau et P. Juillard: Droit international économique, 3éme édition, Dalloz, Paris, 2007. 
Haroun Mehdi : Le régime des investissements en Algérie, à la lumière des conventions franco-algériennes, Litec. Paris. 2000.

Lankarani el- zein Leila : Les contrats d'Etat à l'épreuve du droit international, Collection Droit international, Editions Bruylant, Editions de l'université de Bruxelles, 2001.

Leben Charles : Quelques réflexions théoriques à propos des contrats d'Etat, in, Souveraineté étatique et marchés internationaux. A propos de 30 ans de recherche du CREDIMI. Mélanges en l'honneur de Philippe Kahn. Litec. Année 2000- Volume 20.

Terki Nouredine : La protection conventionnelle de l'investissement étranger en Algérie. Revue algérienne des sciences juridique, économique et politique, Volume 39, 2001.

Revue « ANDI news » bulletin trimestriel.

Site andi.dz 